\title{
Studying Human Tutors to Facilitate Self-Explanation
}

\author{
Amali WEERASINGHE and Antonija MITROVIC \\ Intelligent Computer Tutoring Group \\ Department of Computer Science and Software Engineering \\ University of Canterbury, Christchurch, New Zealand \\ $\{$ acw51,tanja $\} @$ cosc.canterbury.ac.nz
}

\begin{abstract}
This paper reports the first phase of a project with the goal of developing a general model of self-explanation support, which could be used in both open- and closed-ended domains. We studied how human tutors provide additional support to students learning with an existing intelligent tutoring system designed to help students learn database modelling. We report on the findings from this study, which will serve as the basis for defining the model. We also discuss directions for future work.
\end{abstract}

\section{Introduction}

Studies indicate that students acquire shallow knowledge even in the most effective Intelligent Tutoring Systems (ITS) [1]. Self-explanation was shown to facilitate the acquisition of deep knowledge [2]. Several ITSs were enhanced with self-explanation support in domains such as physics [3], mathematics [1], database design [6] and data normalization [5]. With the exception of database design, all these domains are closed-ended, as problem solving is well structured, and therefore self-explanation expected from learners can be clearly defined. Database design is an open-ended task: the final result can be defined in abstract terms, but there is no algorithm to find it. Although the above ITSs were shown to improve student performance, none of these self-explanation models have been used in both open- and closed-ended domains.

Our long-term goal is to develop a model to facilitate self-explanation which can be used in both open- and closed-ended domains. We have chosen Entity-Relationship (ER) modelling as the open-ended domain, and ER-to-relational mapping as a closedended domain. The later task is a well-formed one, and therefore is a deterministic algorithm that students learn in database courses. EER-Tutor [7] and ERM-Tutor [4] are two existing constraint-based tutors. Our goal is to develop a general selfexplanation model that can be used to enhance these systems.

In order to develop a model for self-explanation, we need to consider three basic decisions: when to prompt for self-explanation, what to self-explain and how to obtain self-explanation from learners. As the first step, we conducted a study to observe how students interacted with the EER-Tutor, while providing additional help by a human tutor through a chat interface. Section 2 presents this study. The next section discusses the findings of this study and how they can be incorporated in a self-explanation model. Section 3details the conclusions and the directions of future work section. 


\section{Preliminary Study}

The study was conducted in August 2005 at the University of Canterbury, and involved volunteers enrolled in an introductory database course and professional tutors. The professional tutors will be referred to as tutors, while EER-Tutor as the system hereafter. EER-Tutor provides a problem-solving environment and complements classroom instruction. The version of EER-Tutor used in the study was enhanced with a chat interface, so that the tutors could provide one-to-one feedback to students. We wanted to make the bandwidth between the student and the tutor very similar to that between the student and the ITS. As a result, tutors could observe only the students' interactions with the ITS. Participants interacted with the system in one room and the tutors observed their interactions in another room.

The tutors were not given any specific instructions on providing assistance to students. Student participants were not told that a human tutor was involved in the study. Students also could ask for help through the chat interface or the More Help button in the interface. All interactions were recorded. Students themselves decided when to end the session. All participants filled out a questionnaire on their perceptions about the system and interventions through the chat interface. The tutors were also interviewed to understand their views on the tutoring experience.

\section{Observations and Prototype for the Self-Explanation Model}

Seven students and four professional tutors participated in the study, with at most two students per tutor. The average duration of sessions was 85 minutes $(\mathrm{sd}=20)$. The average number of problems attempted was $11(\mathrm{sd}=5)$, and all the participants completed all the problems attempted. The timing of tutor interventions differed significantly. Some tutors intervened in the first problem in which the student needed help, while in other sessions, the tutors intervened mostly in $4^{\text {th }}$ or the $5^{\text {th }}$ problem. In one situation, the tutor waited until the $19^{\text {th }}$ problem to intervene.

The self-explanation model will be developed on the basis of the findings from this study. The model will decide when and what to self-explain, and how to obtain self-explanations. As all tutors provided delayed feedback, which was well-received by the participants, the model will provide delayed feedback. With delayed feedback, specific guidelines to decide on the timing of interventions need to be incorporated into the model. In the study, delayed feedback was provided in the following situations: (i) the student has been inactive or moving the mouse aimlessly for a predetermined period of a time, (ii) the student has made the same mistake repeatedly or (iii) the student seems to be reacting to feedback without much reflection.

In the first scenario, it will be beneficial to prompt the student to ask a question in order to understand the difficulty in completing the solution, to which the system can respond appropriately. This either requires natural language capabilities or obtaining the response through menu options. For instance, we can ask the student which concept he/she is having difficulties with, and provide a menu for the student to select the concept he/she needs assistance with. As noted in (ii), if the student makes the same mistake repeatedly, it is clear that there is a misconception or gap in his/her knowledge. In such a situation, it will be more beneficial to provide a problemindependent explanation initially. Then the student may need assistance to understand how to apply the domain concept to the current state of the problem. A student seems 
to be reacting to feedback without reflection if he/she makes a single change without reflecting on the other changes that need to be performed as a result. In such situations, the student will be prompted to reflect on other related changes.

The self-explanation model also needs to decide how to prompt learners to selfexplain. As explained earlier, we have seen that human tutors provide problemindependent explanations when there is evidence that a student has difficulty with a domain concept. Later on, the student can be prompted to understand how the corresponding domain concept relates to the current problem state. At other times, the student may have difficulty with the current problem. In such a situation, the student can be guided using a series of prompts ranging from rephrasing feedback, discussing the problem-specific details to providing the answer directly. If the system has natural language processing capabilities, students would be able to specify partial answers and correspond with the ITS in a natural manner.

\section{Conclusions and Future Work}

This research focuses on developing a self-explanation model for both open- and closed-ended domains. As the first step, we conducted a study to observe how tutors help students to solve a problem using the EER-Tutor. In addition to the system's feedback, the students were prompted by human tutors through a chat interface. Although the tutors used different kinds of prompts, all of them provided delayed feedback and guided the students towards the solution without giving the answer directly. According to the questionnaire responses, both timing and content of interventions were well received by the students. They also felt that the help received through the chat interface was very useful for understating mistakes on their own, providing an opportunity for self-explanation and reflection.

The findings from the study are being used to develop the model of selfexplanation, which will be used in the next study with ERM-Tutor to understand its applicability in a closed-ended domain. If necessary, the model will be modified and implemented in both EER-Tutor and ERM-Tutor, which will later be evaluated in authentic class room environments.

\section{References}

1. Aleven, V., Koedinger, K. R., Cross, K. Tutoring Answer Explanation Fosters Learning with Understanding. In: Lajoie, S.P. and Vivet, M.(eds.), AIED 1999, IOS Press, 199-206.

2. Chi, M. T. H. Self-explaining Expository Texts: The dual processes of generating inferences and repairing mental models. Advances in Instructional Psychology, (2000) 161-238.

3. Conati, C., VanLehn, K. Toward Computer-Based Support of Meta-Cognitive Skills: a Computational Framework to Coach Self-Explanation. Artificial Intelligence in Education, 11 (2000) 389-415.

4. Milik, N., Marshall, M., Mitrovic, A. Teaching Logical Database Design in ERM-Tutor. Accepted for publication in ITS2006.

5. Mitrovic, A. The Effect of Explaining on Learning: a Case Study with a Data Normalization Tutor. In: C-K Looi, G. McCalla, B. Bredeweg, J. Breuker (eds) Proc. AIED 2005, 499-506.

6. Weerasinghe, A., Mitrovic, A. Supporting Self-Explanation in an Open-ended Domain. In: Gh.Negoita, M., Howlett, R. J., Jain, L.C. (eds.) Proc. of KES 2004 306-313.

7. Zakharov, K., Mitrovic, A., Ohlsson, S., Feedback Micro-engineering in EER-Tutor. In: Looi, C-K., McCalla, G., Bredeweg, B., Breuker, J. (eds.) Proc. AIED 2005, (2005) 718-725. 